Revista de Comunicación y Salud, 2020, Vol. 10, no 2, pp. 301-321

Editado por Cátedra de Comunicación y Salud

ISSN: 2173-1675

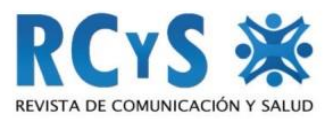

Enviado 15/07/2020

Aprobado 09/09/2020

\title{
RIESGOS PSICOSOCIALES Y ESTRÉS LABORAL EN TIEMPOS DE COVID-19: INSTRUMENTOS PARA SU EVALUACIÓN
}

\section{Psychosocial Risks and Work Stress in Times of COVID-19: Instruments for Its Evaluation}

\author{
Luz Martínez Martínez ${ }^{1}$ \\ Universidad Complutense de Madrid. España \\ luzmar03@ucm.es
}

\section{Resumen}

En el mundo, casi tres millones de personas mueren al año por accidentes o enfermedades relacionadas con el trabajo, más de quinientos sufren enfermedades ocupacionales o lesiones. En 2019, el estrés laboral causó el $30 \%$ de las bajas en España, con un alto coste y reducción de la productividad. La COVID-19 presenta nuevos retos, casi cuatro millones de parados, otros cuatro con un ERTE y una recuperación incierta y lenta que provoca inestabilidad. También nuevos riesgos relacionados con el trabajo y el estrés. Distintos organismos defienden la importancia de la salud y bienestar laboral en todos los aspectos, físicos, mentales y sociales. Ante esta situación es imprescindible la detección de factores psicosociales de riesgo que afectan a la salud en entornos laborales para crear planes de prevención eficaces y adaptados a las situaciones cambiantes. Algunos autores destacan la dificultad de esta evaluación por metodologías insuficientes a nivel conceptual y técnico o faltos de estandarización. El objetivo de este trabajo es identificar distintas herramientas, dimensiones, fiabilidad, validez, análisis psicométrico y uso, que sirvan de referentes para la investigación en PRL. Se realizó un análisis descriptico documental de revisión bibliográfica en buscadores y páginas de organismos especializados. Se ofrecen algunos instrumentos de medición con alta confiabilidad para la detección de factores psicosociales en diversos contextos. Se recomienda complementar estas herramientas con otras que tengan en cuenta otras variables como la incertidumbre, la ansiedad o el estado mental.

Palabras clave: Riesgos psicosociales, Prevención en Riesgos Laborales (PRL), estrés laboral, herramientas de medición, COVID-19, crisis sanitaria.

\footnotetext{
${ }^{1}$ Autora para correspondencia: Luz Martínez Martínez, Universidad Complutense de Madrid, luzmar03@ucm.es
} 
Riesgos psicosociales y estrés laboral en tiempos de COVID-19: instrumentos para su evaluación

\begin{abstract}
In the world, every year almost three million people die from accidents or work-related illnesses, more than five hundred suffer from occupational diseases or injuries. In 2019, job stress caused 30\% of sick leave in Spain, with a high cost and a reduction of productivity. The COVID-19 presents new challenges, almost four million unemployed, another four with an ERTE and an uncertain and slow recovery that causes instability. Also new risks related to work and stress, such as biological contamination, control measures, uncertainty and a high mental burden for workers. Different organizations defend the importance of occupational health and well-being in all aspects, physical, mental and social. Given this situation, it is essential to detect psychosocial risk factors that affect health in the workplace to create effective prevention plans adapted to changing situations. Some authors highlight the difficulty of this evaluation due to insufficient methodologies at the conceptual and technical level or lack of standardization. The objective of this research is to identify different tools, dimensions, reliability, validity, psychometric analysis and use, which serve as references for research in occupational risk prevention. A descriptive analysis of bibliographic review was carried out in searching engines and pages of specialized organizations. Some highly reliable measurement instruments are offered for the detection of psychosocial factors in various contexts. It is recommended to complement these tools with others that introduce other variables such as uncertainty, anxiety or mental state.
\end{abstract}

Keywords: Psychosocial risks, Occupational Risk Prevention, work stress, measurement tools, COVID-19, health crisis.

\title{
Cómo citar el artículo
}

Martínez-Martínez, L. (2020). Riesgos psicosociales y estrés laboral en tiempos de COVID-19: instrumentos para su evaluación. Revista de Comunicación y Salud, 10 (2), 301-321. doi: https://doi.org/10.35669/rcys.2020.10(2).301-321

\section{INTRODUCCIÓN}

Según la Organización Internacional del Trabajo (OIT, 2019), cada año más de 2,8 millones de personas mueren por accidentes laborales o enfermedades relacionadas con el trabajo, 160 millones de trabajadores sufren enfermedades ocupacionales y se producen 374 millones de lesiones no mortales relacionadas con el trabajo estimando el coste de las malas prácticas de seguridad y salud en un 3,94\% del PIB global de cada año. Según la Organización Mundial de la Salud, la salud no sólo se refiere a la ausencia de enfermedad, sino que es "un complejo estado de bienestar en los aspectos físicos, mentales y sociales” (OMS, 1948). El trabajo es fuente de salud, no sólo por la adquisición de un salario que permita comprar bienes, sino porque conlleva una actividad física y mental, un contacto social que permite el desarrollo afectivo, permite sentirse útiles y satisfechos con unos resultados. Además, supone una aportación al bienestar de otros mediante la producción de bienes y de servicios. Sin embargo, el trabajo también puede causar daños a la salud, no sólo a través de accidentes, sino de

Revista de Comunicación y Salud, 2020, Vol. 10, no 2, pp. 301-321 
Riesgos psicosociales y estrés laboral en tiempos de COVID-19: instrumentos para su evaluación

enfermedades profesionales, de la organización del trabajo y de las relaciones laborales, dando lugar a otro tipo de enfermedades como estados de desgaste (burnout) o estrés (OIT, 2019).

En este sentido, la salud laboral se preocupa del bienestar no sólo en la realización del trabajo sino también de sus consecuencias en los planos físicos, mental y social. Es por ello que debe de trabajar, mediante la prevención de lesiones y enfermedades, así como en la promoción de la salud de los trabajadores, ayudando a crear un mercado laboral de calidad (Benavides, Delclós y Serra, 2018). En España, la Ley de Prevención de Riesgos Laborales (PRL) (Ley 31/1995) obliga a las empresas a prevenir las lesiones y enfermedades, esto concierne a actuaciones de prevención sobre las condiciones de trabajo, no sólo estructurales, ambientales y psicosociales; sino también actividades de promoción de la salud que eduquen a los trabajadores en el cuidado de su propio bienestar. Por lo tanto, la promoción de la salud en el lugar de trabajo debe de hacer un abordaje integral que incluya también los factores psicosociales y la organización del trabajo (Sjöberg, 2017). Esto es especialmente importante en situaciones de crisis como la actual con la COVID-19 en la que los trabajadores y el mercado laboral se han visto afectados de varias maneras (factores macroeconómicos, endeudamiento, cierre de negocios, etc).

\subsection{Estrés laboral y riesgos psicosociales}

Si bien el estrés es una reacción natural de nuestro organismo que facilita la respuesta y adaptación al entorno, imprescindible para la propia supervivencia, y que ayuda a los seres humanos a desempeñarse eficazmente en sus actividades (Ivancevich y Matteson, 1989; Peiró, 2005), es importante mantener los niveles de estrés moderados para disfrutar de sus beneficios tanto para la salud como para el rendimiento. Cuando nos estresamos, nuestro sistema nervioso autónomo responde a un estado de alarma con adaptaciones psicofisiológicas, el corazón bombea más sangre hacia las extremidades, el ritmo cardiaco se acelera, las pupilas se dilatan y el cerebro segrega más cortisona (Sandín, 2003). Lo normal es que vivamos en estados intermitentes de estrés y que los niveles se normalicen cuando el estímulo o estresor desaparece, en este caso sería un estrés positivo o eutrés (Selye, 1960). Si éste persiste, el organismo desarrollará resistencia lo que le permitirá lidiar con las tensiones durante un tiempo, pero a costa de disminuir sus reservas bioquímicas, dando lugar al agotamiento, convirtiéndose en estrés negativo o distrés. Cuando el estímulo estresor persiste y el estrés se vive como un estado permanente, el organismo no se recupera, y en dosis altas y de manera continuada se convierte en estados nocivos para la salud. Este estado, no sólo aumenta los riesgos de sufrir accidentes físicos en el trabajo por falta de atención o desorientación, sino que producen también emociones negativas, lo que conlleva a una propensión de la activación del sistema autónomo y neuroendocrino (Siegrist, 1996).

El estrés laboral conlleva unas respuestas psico-fisiológicas por la percepción amenazante de un factor de riesgo (Sauter, Murphy y Levi, 1998) o factores psicosociales de riesgo que afecta a la salud de los trabajadores y al funcionamiento

Revista de Comunicación y Salud, 2020, Vol. 10, no 2, pp. 301-321 
Riesgos psicosociales y estrés laboral en tiempos de COVID-19: instrumentos para su evaluación

empresarial (Jiménez y León, 2010), es un desequilibrio entre la demanda y la capacidad de respuesta (García-Herrero, Mariscal, Gutiérrez y Ritzel, 2013). Este estrés provoca sensación de angustia, cansancio e intranquilidad, irritabilidad, dificultades para tomar decisiones, e incluso problemas físicos como cardiopatías, aumento de la tensión arterial, trastornos digestivos y musculo-esqueléticos entre otros (Leka, Griffiths y Cox, 2004). Aunque el estrés laboral se ha considerado con frecuencia como una experiencia subjetiva de una persona derivada de la percepción de tener que tratar con demandas excesivas o amenazantes difíciles de controlar, éste no afecta sólo a la salud y bienestar físico de las personas, sino que repercute en su actividad en el trabajo y relación con el entorno. La exposición a un ambiente laboral perjudicial puede conllevar un mayor absentismo, menor productividad, problemas psicosomáticos y afectivos, incremento de la práctica de hábitos adictivos como beber y fumar, y la reducción de otras saludables como la actividad física (Fernández, Fernández y Siegrist, 2005; Sandín, 2003). A nivel cognitivo, provoca sensación de preocupación, indecisión, baja concentración, desorientación, mal humor y sentimientos de falta de control (López et al., 2004)

\subsection{Qué nos produce estrés y cuál es su relación con el trabajo}

Según la "Teoría transaccional" de Lazarus y Folkman (1986), además de una estructura biológica, los individuos están determinados por el extracto social y las experiencias vividas. Factores individuales y sociales intervienen en la evaluación sobre situaciones y su significado, influyendo en la construcción de valores, compromisos y creencias. Esta evaluación cognitiva comprende una interpretación del individuo de una situación como amenazante o peligrosa para sí mismo. Es por esto, que una situación no afecta de la misma forma a todos, ya que entran en juego características personales como los valores, compromisos, creencias, forma de afrontamiento y recursos individuales. En relación al estrés laboral, el "Modelo demanda-control-apoyo social" (Karasek, 1979, Karasek y Theorell, 1990) es una de las perspectivas más utilizadas. Inicialmente, este modelo planteaba que entre las principales fuentes de estrés se encuentran dos factores: las demandas psicológicas del trabajo (presión de tiempo, carga mental y conflictos de roles) y el control que se tiene del mismo (trabajo monótono, ejercicio de competencias, autonomía y uso de capacidades). Más tarde Jeffrey Johnson (1986) añade la variable moderadora de apoyo social (interacción con compañeros y supervisores) ya que puede incrementar la habilidad para hacer frente a una situación de estrés mantenido. Bajo estas premisas, el estrés puede analizarse a través de los estresores (riesgo de enfermedad relacionada con el estrés) y el grado en el que el trabajador puede tomar decisiones acerca de su trabajo (comportamiento activo/pasivo). Un trabajo con altos niveles de demandas de control y apoyo social sería un trabajo activo sin ser estresante, promoviendo la motivación, el aprendizaje y el crecimiento personal. Una situación con altas demandas, bajo control y poco apoyo sí que sería estresante al tener la percepción de no poder responder a esas demandas, en este caso se producen tensiones psicológicas como fatiga, depresión, ansiedad y enfermedades físicas (Johnson y Hall, 1988).

Revista de Comunicación y Salud, 2020, Vol. 10, nº 2, pp. 301-321 
Por otro lado, la "Teoría del ajuste persona-entorno" (P-E) contempla la forma en que la interacción de las características individuales y del trabajo influyen en el bienestar del trabajador (Dawis y Lofquist, 1984; Pervin, 1967; Luceño, Martín, Jaén y Díaz, 2005) como determinante para la comprensión de las reacciones cognitivas, afectivas y de comportamiento. En este modelo, un mal ajuste se daría por el desequilibrio entre las exigencias del puesto y las necesidades del trabajador de utilizar sus capacidades y habilidades. Este modelo incorpora el concepto de frustración como una sensación moderadora del estrés laboral. Siguiendo con esta línea, Siegrist (1996) postula el "Modelo Desequilibrio Esfuerzo-Recompensa" (DER) que analiza el equilibrio entre los costes o esfuerzos del trabajador (demandas del trabajo, motivación e implicación) y las ganancias o recompensas que recibe a cambio (monetaria, apoyo organizacional y de seguridad, como promoción y estabilidad). Para este autor, las exigencias influirán en la autorregulación sobre la autoestima, auto-eficacia y sensación de formar parte del grupo. Por lo tanto, cuando existe desequilibrio entre el esfuerzo y la recompensa, pueden aparecer sentimientos de amenaza, ira, depresión y desmoralización. Este modelo no sólo considera variables estructurales del ambiente de trabajo, sino que incorpora variables personales. Destaca la importancia de la interpretación por parte del sujeto que puede hacer que esa relación entre el esfuerzo y la recompensa no sea real, la necesidad de control como una característica de personalidad y la implicación (estilo personal de afrontamiento).

La percepción de la realidad de cada persona está basada en la interpretación que haga, a su vez, esta interpretación estará influenciada por experiencias pasadas, patrones de creencias y valores, actitudes y emociones provocadas tanto en el entorno laboral como otros aspectos de la vida. Por eso es importante distinguir ciertas características del sujeto en las percepciones entre esfuerzo y recompensa. Por ejemplo, un individuo con un patrón motivacional con un compromiso alto y alta necesidad de aprobación es más vulnerable a la frustración y a la tensión al exponerse más a altas demandas de trabajo o exagerar sus esfuerzos. También provocará un desequilibrio tener pocas oportunidades para cambiar de empleo, la exposición al desempleo y a cambios socioeconómicos rápidos (Fernández et al., 2005; Luceño et al., 2005). Estas perspectivas han incorporado el concepto de riesgo psicosociales en relación con el trabajo.

\subsection{Importancia de evaluar el estrés laboral}

Las transformaciones en el mundo laboral (trabajos automatizados, cambios demográficos, exigencias temporales, intensidad) generan la aparición de nuevos riesgos psicosociales relacionados con el estrés laboral crónico y al síndrome del trabajador quemado o "burnout" (OIT, 2019) sobre todo en profesionales que dan servicio a otras personas (EU-OSHA, 2019). De hecho, para 2022 este trastorno estará reconocido por la OMS como enfermedad relacionada con el trabajo al ser incluido en la actualización de la "Clasificación Internacional de Enfermedades y Problemas de Salud Conexos" (CIE-11). Se trata de dar visibilidad a la dolencia y facilitar la gestión de bajas e incapacidades por estrés laboral. Para ello se tendrán en cuenta tres elementos: "una sensación de agotamiento", "cinismo o sentimientos negativos relacionados con su

Revista de Comunicación y Salud, 2020, Vol. 10, nº 2, pp. 301-321 
Riesgos psicosociales y estrés laboral en tiempos de COVID-19: instrumentos para su evaluación

trabajo" y una "eficacia profesional reducida". Este manifiesto supone un paso hacia la recomendación de la Comisión Mundial sobre el Futuro del Trabajo para que la seguridad y la salud en el trabajo sean reconocidas como un principio y un derecho fundamental.

Según datos de la Tesorería General de la Seguridad Social (TGSS, 2020), en 2019 el estrés laboral fue el causante del 30\% de las bajas laborales en España, el $40 \%$ de los trabajadores y más de la mitad de los autónomos declararon estar estresados, provocando que fueran un 60\% menos eficientes en el trabajo. Según Eurostat (2019), España es el tercer país europeo con mayor estrés laboral con casi 500.000 afectados. En el 2019, se percibe un mayor descontento con el horario laboral y las relaciones con los compañeros y supervisores causando un "ambiente deprimente" y "menos productividad" (Cigna, 2019). Un 72\% afirmaba que donde trabaja no se le proporciona ningún programa de salud y bienestar. Lo significativo de esta información es que el $42 \%$ de los españoles cree que estos programas se refieren a políticas centradas únicamente en el cuidado del bienestar físico, y no mental, mostrando un alto desconocimiento al respecto. Además, sólo el $7 \%$ de los que tienen un programa de bienestar participan en él. La Encuesta Europea en Empresas sobre Riesgos Nuevos y Emergentes (EU-OSHA, 2019) en su último informe recoge estas tendencias a nivel europeo, muestra que la conciencia sobre los riesgos psicosociales derivados del trabajo sigue siendo baja frente a riesgos más físicos como los musculoesqueléticos. También son más complicados de manejar, sobre todo porque los trabajadores renuncian a hablar abiertamente de estos temas y por falta de conciencia de la gerencia y de los propios trabajadores (Figura 1).

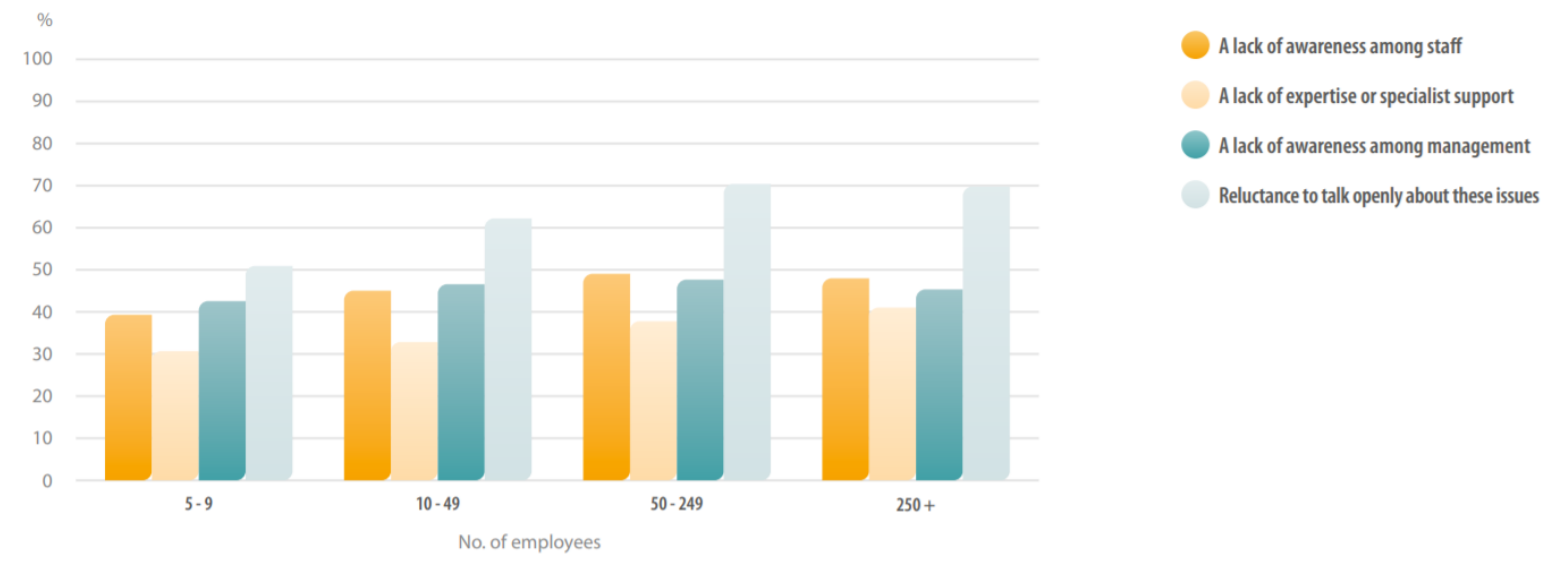

Figura 1. Factores que hacen que los riesgos psicosociales sean más difíciles de manejar, por tamaño (número de empleados)

Fuente: EU-OSHA (2019). Encuesta de la Agencia Europea para la Seguridad y la Salud en el trabajo ESENER 2019 "What does it tell us about safety and health in Europe's workplaces?". https://osha.europa.eu/es/publications/esener-2019-policy$\underline{\text { brief/view }}$

Podemos observar que existe una falta de conocimiento y concienciación tanto de empleados como de empleadores. Aunque muchas veces se asocia la gestión de la

Revista de Comunicación y Salud, 2020, Vol. 10, № 2, pp. 301-321 
Riesgos psicosociales y estrés laboral en tiempos de COVID-19: instrumentos para su evaluación

seguridad y salud con el rendimiento, el informe muestra que las empresas están principalmente motivadas a implantar estas medidas por obligación legal, por cubrir exigencias de trabajadores y por evitar posibles multas por inspección laboral. Sin embargo, desde el 2014 ha habido un descenso del $8 \%$ en el número de estas inspecciones. Las evaluaciones de riesgo periódicas no se realizan por la percepción de que ya se conocen todos los riesgos (83\%) y porque "no hay mayor problema" $(80 \%)$, percepciones a su vez relacionadas con la ausencia de conocimiento de factores de riesgo. Además, existen otras variables que dificultan la gestión de estos programas como la complejidad de las obligaciones legales, el papeleo o la falta de tiempo del personal.

Para la buena gestión de la prevención es importante desarrollar planes de $P R L$ que permitan estructurar y comunicar correctamente, en función de objetivos y necesidades, desarrollar el diálogo continuo y la escucha activa que tenga en cuenta la opinión y percepciones de los trabajadores, así como identificar posibles carencias, enfocando siempre la prevención como una actividad participativa. En este sentido, los empleadores o responsables deben hacer uso de distintas herramientas de evaluación validados (cuestionarios, grupos focales, entrevistas, observación, etc) que permitan identificar factores de riesgo o estresores para la aplicación de medidas más útiles (Artazcoz y Molinero, 2004; Ortiz, 2010; Landsbergis, Schnall, Pickering y Schwartz, 2002). La OMS (Leka et al., 2004) insta a los estados miembros a generar planes de salud ocupacional evaluables para promover el bienestar de los trabajadores. Según el mismo informe, algunas de las medidas más utilizadas por las empresas para prevenir riesgos psicosociales son: permitir a los empleados tomar decisiones sobre cómo hacer su trabajo, reorganización del trabajo para reducir demandas de puesto y presión, asesoramiento confidencial, entrenamiento en resolución de conflictos e intervención si la jornada es larga o se trabajan horas irregulares. Como muestra la Figura 2, las aplicaciones de estas medidas varían en función del tamaño de la empresa.

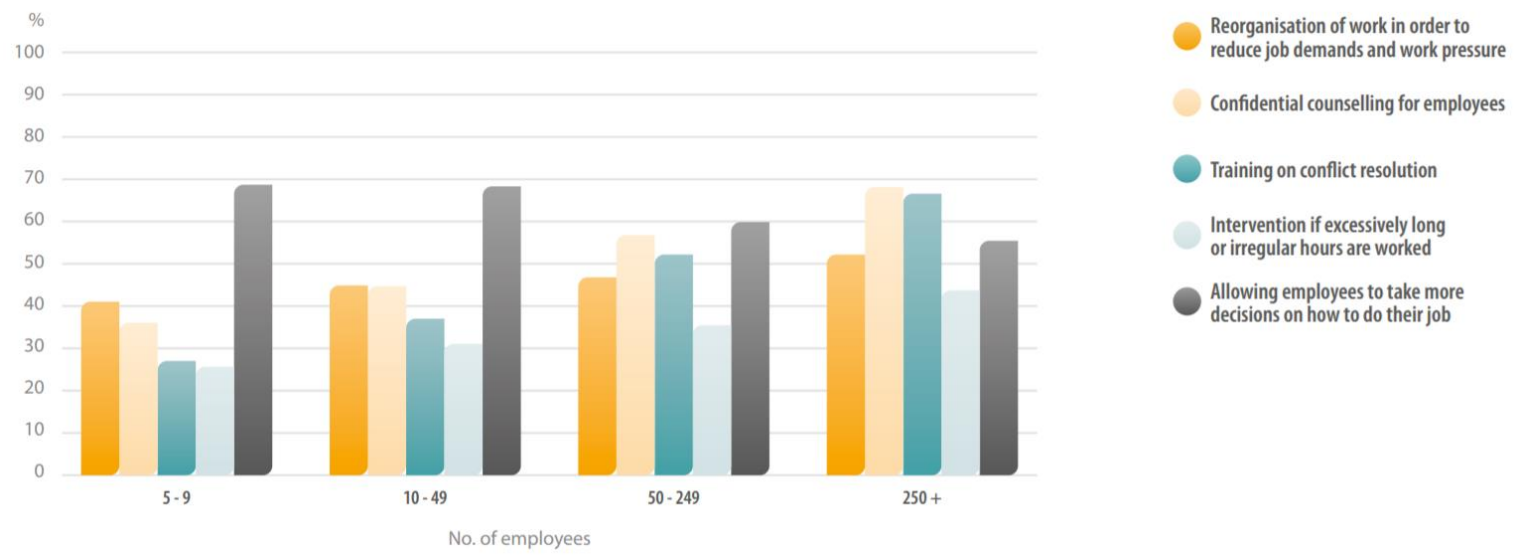

Figura 2. Medidas en lugares de trabajo para prevenir riesgos psicosociales, por tamaño (número de empleados), 2019

Fuente: EU-OSHA (2019). Encuesta de la Agencia Europea para la Seguridad y la Salud en el trabajo ESENER 2019 "What does it tell us about safety and health in Europe's workplaces?". https://osha.europa.eu/es/publications/esener-2019-policy$\underline{\text { brief/view }}$

Revista de Comunicación y Salud, 2020, Vol. 10, oㅡ 2, pp. 301-321 
Riesgos psicosociales y estrés laboral en tiempos de COVID-19: instrumentos para su evaluación

\subsection{Prevención en tiempos de COVID-19}

La situación de la COVID-19 ha supuesto un golpe y una transformación en numerosas empresas con grandes repercusiones sobre los ingresos y los empleos, una situación catalogada ya como la peor crisis mundial desde la Segunda Guerra Mundial (OIT, 2020). Según el informe del observatorio de la Organización Internacional del Trabajo "La COVID-19 y el mundo del trabajo" (2020), alrededor de 436 millones de empresas de los sectores más afectados (hostelería y restauración, industria manufacturera, comercio, actividades inmobiliarias y actividades comerciales) experimentarán grandes perturbaciones. El $68 \%$ de la fuerza de trabajo mundial se localiza en países que han cerrado lugares de trabajo por confinamiento, exponiéndolas a un riesgo elevado de insolvencia. Pero, además, estas empresas tendrán que prepararse para una recuperación incierta y lenta. Con necesidad de realizar ajustes que garanticen ambientes de trabajo seguros y medidas dirigidas a proteger tanto a las empresas como a los trabajadores.

Esta situación no sólo ha supuesto una crisis de salud, estas medidas han afectado directamente al mercado laboral provocando una profunda inestabilidad e inquietud. Según datos del Ministerio de Trabajo y Economía Social de España (2020) en el mes de marzo y abril se registró un descenso en el número de afiliados a la Seguridad Social de 947.896, subiendo la cifra de parados a 3,7 millones. En abril también se registró un descenso del número de empresas inscritas, un $-7,9 \%$ respecto al mismo periodo en 2019. De los afiliados, cerca de 4 millones (casi el 25\%) tienen una suspensión total o parcial por Expedientes de Regulación Temporal de Empleo (ERTE) y más de un millón de autónomos han solicitado la ayuda por cese de actividad. Aquellas empresas que pueden realizar su actividad por su naturaleza o que han ido abriendo paulatinamente según las fases marcadas por el Gobierno, deben adaptar sus espacios y protocolos con el fin de salvaguardar la salud tanto de trabajadores como de clientes. Algunas de estas medidas consisten en mantener distancias de seguridad, higiene de espacios, manos y calzado, uso de mascarillas, guantes y pantallas de protección, e incluso la cuarentena de productos tocados por clientes (INSST, 2020; EU-OSHA, 2020). Los empresarios son los responsables de asegurar tales medidas a los trabajadores, pero en el caso del sector servicios con atención al público, son también los propios trabajadores los que controlarán que los clientes cumplan con las medidas de prevención.

Por lo tanto, los trabajadores se encuentran ante nuevos riesgos o peligros relacionados con el trabajo y el estrés, como el de contaminación biológica, no por el trabajo en sí sino por la exposición a la pandemia. Tareas que se vuelven desagradables por miedo al contagio por exposición a otros, falta o ineficacia de medidas de control, el propio miedo social y la incertidumbre en su puesto de trabajo (Leka et al., 2004). Esta situación también supone una carga mental para los trabajadores que deben interpretar continuamente lo que sucede a su alrededor en el medio material y social. Esto aumenta sus demandas de concentración y atención, se produce gran cantidad y dispersión de información sobre la regulación, normas y prevención que deben asimilar. De hecho, la OMS ya advierte de la necesidad de una

Revista de Comunicación y Salud, 2020, Vol. 10, nº 2, pp. 301-321 
Riesgos psicosociales y estrés laboral en tiempos de COVID-19: instrumentos para su evaluación

mayor inversión en salud mental ante el riesgo de un aumento drástico de enfermedades psíquicas (OMS, 2020). Factores como el aislamiento social, el miedo al contagio, la pérdida de familiares, la pérdida de ingresos y, en muchos casos, de empleo hacen que los efectos de la COVID-19 sobre la salud mental sean muy elevados.

\section{OBJETIVOS}

Ante esta situación es crítico diseñar planes de salud con programas preventivos que promuevan el bienestar de los trabajadores, para ello es necesario estudiar los distintos factores del estrés psicológico y social. Desde varios organismos como la Agencia Europea para la Seguridad y la Salud en el Trabajo (EU-OSHA) o el Instituto Nacional de Seguridad e Higiene (INSHT) se recomiendan varias herramientas de evaluación y monitoreo que permiten identificar posibles riesgos psicosociales o estresores que influyen en la producción de estrés en el trabajo. Sin embargo, algunos autores remarcan la dificultad de esta evaluación mediante metodologías tradicionales, insuficientes a nivel conceptual y técnico o carentes de procesos de estandarización recomendando la incorporación de ítems orientados a capturar la experiencia de los trabajadores también en su dimensión subjetiva y psicosocial (Blanch, Sahagún y Cervantes 2010; Olmos, Cebollero y Carrejón, 2008; INSHT, 2004; Tejada y Gómez, 2009). En esta investigación se exponen algunos de los cuestionarios estandarizados más utilizados con el objetivo de unificar criterios a la hora de analizar los riesgos psicosociales en el trabajo y que sirvan de referentes para la investigación en PRL. Conocer las distintas dimensiones y su aplicación permite el análisis de los posibles estresores en el ámbito laboral y con ello, un mejor diseño de medidas de prevención.

\section{METODOLOGÍA}

Para el estudio se llevó a cabo un análisis descriptico documental de revisión bibliográfica de las herramientas utilizadas para la evaluación de riesgos psicosociales en el trabajo entre los años 2009-2020 en distintos buscadores y bases de datos como Google scholar, fuentes bibliográficas UCM, WOS, Science Direct, SciELO, Dialnet, DOAJ, JOEM y páginas oficiales del Ministerios de Trabajo, INSHT, OMS, OIT y EUOSHA. Para ello se utilizaron los valores "estrés laboral", "estrés ocupacional", "riesgos psicosociales", "occupational stress", "work stress", "psychosocial risks" y "working conditions". Se procedió a la lectura y revisión de los trabajos buscando las herramientas validadas tanto por instituciones como por diferentes investigaciones, para ello se tuvieron en cuenta los trabajos de adaptaciones idiomáticas como de aplicación en diferentes sectores (salud, educación, construcción, manufacturera, etc) analizando los antecedentes de investigación, índice de fiabilidad, pruebas de validez y análisis psicométrico de los ítems (Lluís y Navarro, 2006).

\section{DISCUSIÓN}

En la actualidad existen diferentes tipos o categorías de cuestionarios en relación a la PRL y los riesgos psicosociales (Blanch et al., 2010). Los que brindan un enfoque

Revista de Comunicación y Salud, 2020, Vol. 10, oㅡ 2, pp. 301-321 
Riesgos psicosociales y estrés laboral en tiempos de COVID-19: instrumentos para su evaluación

general sobre las condiciones de trabajo desde la dimensión objetiva de los distintos factores de riesgo, pero que no tienen en cuenta las realidades estructurales de la organización ni de la personalidad, un ejemplo es la encuesta de Calidad de Vida en el Trabajo (Villalobos, Vargas, Escobar, Jiménez y Rondón, 2010). Los que analizan algún efecto particular de la exposición a factores psicosociales como el burnout, satisfacción laboral, acoso, etc. Como la Escala de Desgaste Ocupacional (Uribe-Prado, 2010), el Inventario de Violencia y Acoso Psicológico en el Trabajo (Moreno, Beltrán, Serrano, Chávez y Estrada, 2006). Por último, están los cuestionarios de autoinforme imprescindibles para recoger y analizar la percepción de los trabajadores y los procesos que generan estrés. Éstos consideran la relación entre varias dimensiones y factores, tanto individuales como sociales y laborales. A continuación, se presentan varias opciones de este tipo de cuestionarios con validez como herramientas predictivas que permiten relacionar dimensiones del entorno laboral con problemas de salud. (Bourbonnais, Comeau y Vézina, 1999; Karasek, 1979; 1996; Siegrist, 1996; 2004; 2010).

\subsection{Cuestionario demanda-control- apoyo social (DCS)}

Basado en el modelo inicial de demanda-control (Karasek 1979; Karasek y Theorell, 1990). La demanda sería la combinación de tres dimensiones: cantidad y tipo de demanda; control sobre las tareas que se realizan; y apoyo social como posible intensificador de la reacción estresante (Johnson, 1986; Johnson y Hall, 1988). Permite la identificación de niveles de estrés laboral en cuatro niveles desde baja tensión a alta. Una situación de alta tensión se daría cuando las demandas psicológicas exceden su control sobre el trabajo y además existe un bajo apoyo social vs baja tensión, con bajas demandas y alto control. Los niveles intermedios se identificarían como trabajos activos (alta demanda y alto control) y pasivos (bajas demandas y bajo control). Se trata de un cuestionario de 22 ítems en escala Likert de 4 puntos, adaptado al español y validado en diferentes investigaciones. Por ejemplo, esta herramienta fue utilizada por Lazcano y su equipo (1996) en su investigación sobre la relación entre tensión laboral, satisfacción laboral y trastornos psicológicos en el sector sanitario mostrando la relación entre tensión laboral y satisfacción, así como de la tensión y los trastornos. También en el sector sanitario, García, Rísquez, Fernández y Roche (2018) utilizaron este modelo para la identificación de factores de estrés laboral en el personal de enfermería encontrando un escaso control sobre el trabajo, una alta exigencia en aprendizaje y multitarea y un bajo apoyo social, sobre todo de superiores, lo que podría favorecer la aparición de estrés.

En otro estudio sobre la relación del modelo y la creatividad docente (Gallegos, Iturrizaga y Salinas, 2014) encontraron una relación positiva entre control, apoyo social y creatividad. Aunque se encontraron altos niveles de estrés, con grandes demandas, también se observó un alto control categorizando la actividad del docente como un trabajo activo, más que como de alta tensión. También se encontró una relación positiva entre el estrés y la creatividad, mostrando que en cierto grado de estrés favorece la creatividad en el trabajo. Más recientemente, en referencia a la cultura organizacional y el impacto del trabajo digital, Castiblanque y Palomares (2020)

Revista de Comunicación y Salud, 2020, Vol. 10, nº 2, pp. 301-321 
Riesgos psicosociales y estrés laboral en tiempos de COVID-19: instrumentos para su evaluación

comprobaron que la existencia de una intensificación laboral, un menor control y bajo apoyo social derivan en mayores niveles de tecnoestrés, tecnoansiedad o ciberacoso en aquellos trabajadores digitales vs no digitales, y un menor grado de protección.

\subsection{Cuestionario de Desequilibrio Esfuerzo-Recompensa (DER)}

El cuestionario de Desequilibrio Esfuerzo-Recompensa (DER) es la versión española del cuestionario alemán Efford-Reawrd Imbalance Questionnaire (ERI). Basado en el modelo de Esfuerso-Recompensa de Siegrist (1996) analiza la relación de intercambio de la función laboral (esfuerzo) en el proceso de reciprocidad social (gratificaciones). Permite identificar reacciones estresantes en el trabajo como aquellas que suponen un alto coste o esfuerzo y un bajo beneficio o recompensa, combinando información contextual de la estructura de recompensa, características del trabajo y evaluativa de los estados de superación del estrés. La discrepancia entre esfuerzo y recompensas sería un determinante de las reacciones estresantes y sus efectos. Dispone de varias versiones, la extensa constaba de 23 ítems, sin embargo, ha sufrido modificaciones para poder ser aplicado también a autónomos o propietarios de pequeñas empresas (Siegrist, Li y Montano, 2014) quedando un cuestionario de 22 ítems en escala Likert de 4 puntos. Estos ítems están agregados a 3 escalas principales: esfuerzo extrínseco (escala laboral, incremento de demandas y exigencias físicas), recompensa (adecuación salario, posibilidades de promoción, seguridad laboral y estima recibida) y sobreimpresión (esfuerzo intrínseco). Las preguntas se refieren al puesto actual ocupado o más reciente.

Trabaja sobre dos dimensiones, el esfuerzo extrínseco o percepciones del propio trabajador sobre el esfuerzo realizado y la recompensa profesional, y el esfuerzo intrínseco evaluando el grado de implicación del trabajador en su puesto de trabajo. En la investigación de Tirado, Llorente y Topa (2019) sobre el desequilibrio esfuerzorecompensa percibido en las quejas de salud en médicos comprobaron un efecto mayor en individuos con sobreimplicación alta o media. Para analizar la relación del desequilibrio entre esfuerzo - recompensa con el malestar mental algunas investigaciones complementan el DER con el cuestionario de salud general (General Halth Questionnaire, GHQ-28), estos trabajos han encontrado una asociación entre la presencia de estrés con un mal estado de salud mental, síntomas de depresión y de tipos somáticos, y el sobre compromiso con ansiedad e insomnio (Canepa, Briones, Pérez, Vera y Juárez, 2008). En esta misma línea, Tsutsumi, Kayaba, Theorell y Siegrist (2001) demostraron que un desequilibrio entre esfuerzo y recompensa y el exceso de compromiso se relacionaban con la depresión. Algunas investigaciones también se han centrado en analizar la relación entre estrés en el trabajo y síntomas de enfermedad cardiovascular, hipertensión y colesterol (Siegrist, 2010; Kivimäki et al., 2002; Peter, Geißler y Siegrist, 1998).

\subsection{CoPsoQ (ISTAS21, PSQCAT21)}

Método para la evaluación y la prevención de los riesgos psicosociales. Elaborado por el Instituto Nacional de Salud Laboral de Dinamarca (AMI) permite adaptarse a los

Revista de Comunicación y Salud, 2020, Vol. 10, nº 2, pp. 301-321 
Riesgos psicosociales y estrés laboral en tiempos de COVID-19: instrumentos para su evaluación

cambios en el mundo de trabajo y se puede aplicar a cualquier puesto e identificar los riesgos psicosociales en las empresas y las áreas a mejorar para reducirlos (Kristensen, 2000). Está basado en el modelo en la Teoría General de Estrés e integra las dimensiones de los modelos Demanda - Control - Apoyo social de Karasek y Therorell (1989), y Desequilibrio Esfuerzo - Recompensa (ERI) de Siegrist (1990), asume también la teoría de la doble presencia, que tiene que ver con la necesidad de responder al trabajo doméstico y al asalariado (Balbo, 1994). El AMI cedió los derechos al Instituto Sindical de Trabajo, Ambiente y Salud de España, creando grupos de trabajo para la versión en castellano junto con el Instituto Nacional de Seguridad e Higiene en el Trabajo y otras administraciones, dando lugar al "ISTAS21 COPSOQ" (Moncada, 2004; Moncada, Llorens, Navarro y Kristensen, 2005).

Existen varias versiones en función del tipo de empresas donde se utiliza: versión corta para empresas de menos de 25 trabajadores, versión mediana para más de 25 trabajadores y una más extensa para su uso por personal investigador. La versión media contempla 21 dimensiones distintas abarcando un gran espectro agrupadas a su vez en 5 grupos: Exigencias psicológicas (exigencias cuantitativas, cognitivas, emocionales, exigencias de esconder emociones y exigencias sensoriales), Influencia y desarrollo de habilidades (influencia en el trabajo, posibilidades de desarrollo, control sobre el tiempo de trabajo, sentido del trabajo e integración en la empresa), Apoyo social en la empresa y calidad de liderazgo (previsibilidad, claridad de rol, conflicto de rol, calidad de liderazgo, refuerzo, apoyo social, posibilidad de relación social y sentimiento de grupo), Compensaciones (inseguridad y estima) y doble presencia (doble presencia).

El CoPsoQ (ISTAS21) es una metodología de utilización pública y gratuita utilizada en numerosas investigaciones sobre el estrés laboral. En el análisis de los riesgos psicosociales en profesores universitarios (García, Iglesias, Saleta y Romay, 2016) se detectó que es un trabajo activo, con posibilidades de desarrollo y seguridad laboral, pero con mucha exigencia psicológica, poca estima y apoyo social lo que puede derivar en estresores desfavorables para la salud. En relación a trabajadores sanitarios de atención primaria y hospitalaria (García-Rodriguez, Gutierrez-Bedmar, Bellón-Saameño, Muñoz-Bravo y Navajas, 2015) se pudo comprobar que es un sector con altas exigencias psicológicas cognitivas, emocionales y sensoriales, mostrando todos síntomas de estrés percibido, sobre todo los facultativos de atención primaria. En su investigación sobre gestión de tiempo en la empresa, Cladellas (2008) comprobó que la poca posibilidad de gestión de tiempo se relaciona con más insatisfacción con su trabajo, una peor salud y mayores síntomas de estrés.

\subsection{Escala de Estrés Laboral de la OIT-OMS}

Está sustentada por Ivancecich y Matteson (1989) y con una confiabilidad de 0.966 según el alpha de Cronbach. Es autoadministrable pudiéndose aplicar de manera individual o grupal. Consta de 25 ítems en una escala Likert de 7 puntos, relacionados con la estructura y clima organizacional, tecnología, influencia del líder, territorio, cohesión y respaldo del grupo de trabajo. Para ello los autores identificaron y

Revista de Comunicación y Salud, 2020, Vol. 10, nº 2, pp. 301-321 
Riesgos psicosociales y estrés laboral en tiempos de COVID-19: instrumentos para su evaluación

clasificaron los antecedentes de la situación de estrés como estresores intraorganizacionales (estresores de nivel individual, grupal, organizacionales y del ambiente físico de trabajo) y extraorganizacionales (factores familiares, políticos, sociales y económicos que inciden sobre el individuo). La identificación de los estresores de orden organizacionales, que pueden generar dificultades en el desempeño laboral e interrelación entre los trabajadores, permite implementar medidas de prevención y control de estrés. La herramienta permite predecir las fuentes de riesgos psicosociales y se ha utilizado para el estudio del estrés en diferentes sectores (tipos de ocupación) como administrativos en instituciones policiales (Díaz y Tinoco, 2011), asesores telefónicos (Tunanñaña, 2013), e industria (Chacín-Almarza, CorzoÁlvarez, Rojas-González y Corzo-Ríos, 2002). Estos trabajos muestran la relación del estrés con múltiples factores, destacan por ejemplo el efecto estresor del cambio tecnológico que necesita de herramientas por parte de la empresa, trabajos automatizados y actualización por parte de los empleados (Peiró y Rodríguez, 2008; Díaz y Tinoco, 2011); o de la percepción negativa sobre la influencia del líder. También se ha encontrado diferente percepción de estrés en función de la edad, siendo mayor en grupos de más edad y también en aquellos con mayor antigüedad laboral (ChacínAlmarza et al., 2002).

\section{CONCLUSIONES}

Aunque el estrés laboral está reconocido a nivel mundial como una enfermedad derivada del trabajo donde intervienen determinados riesgos psicosociales (OIT, 2019; OMS, 1948; Siegrist, 1996; Karasek, 1979), y con serias consecuencias económicas, organizacionales y de salud para el mercado laboral (TGSS, 2020; Cigna, 2019; OMS, 2020) existe todavía mucho trabajo en la concienciación de su importancia tanto de empleados como empleadores (EU-OSHA, 2019). Por un lado, estas problemáticas se hacen más difíciles de gestionar por la preferencia de no hablar de ello en público y por propio desconocimiento, por otro lado, aquellas empresas que implantan alguna medida no lo hacen en busca de una mejora de salud sino por obligaciones legales o evitar sanciones. Esto muestra la necesidad de diseñar más campañas informativas de salud y prevención en los lugares de trabajo y de estrategias que faciliten la comunicación dentro de la organización.

La situación de la COVID-19 ha supuesto un tremendo impacto en el trabajo provocando una crisis profunda que ha puesto en alto riesgo a empresarios y empleados (OIT, 2020; TGSS, 2020). Además de las pérdidas producidas por el confinamiento, tanto empresas como trabajadores se deben adaptar a un nuevo escenario en convivencia con la COVID-19 y con las distintas medidas sanitarias como distanciamiento físico, higiene de espacios, mascarilla y pantallas de protección. Esta situación de riesgo demanda: 1) un aumento de la concentración, atención y asimilación de información por parte de los trabajadores, y 2) una sobrecarga de responsabilidad al ser, en muchos casos, los propios trabajadores los encargados de asegurar que los clientes cumplan con las medidas de seguridad adoptadas. Esto supone una gran carga mental que puede desencadenar en altos niveles de estrés con su consecuente perjuicio en la salud. Nos encontramos entonces que ante esta crisis es urgente el

Revista de Comunicación y Salud, 2020, Vol. 10, nº 2, pp. 301-321 
Riesgos psicosociales y estrés laboral en tiempos de COVID-19: instrumentos para su evaluación

diseño de programas preventivos y aplicación de medidas que permitan realizar ajustes y que garanticen ambientes de trabajo seguros, con estrategias dirigidas a proteger tanto a las empresas como a los trabajadores.

Para el diseño de estas medidas de gestión y prevención en COVID-19, se hace imprescindible tener en cuenta los distintos factores del estrés psicológico y social. Con este fin, existen distintos cuestionarios o escalas estandarizados que permiten identificar y monitorear los posibles riesgos psicosociales que influyen en la producción de estrés en el trabajo (Karasek 1979; Johnson y Hall, 1988; Ivancecich y Matteson; 1989; Karasek y Theorell, 1990; Siegrist, 1996; Siegrist et al., 2014; Moncada et al., 2005). Estas herramientas han demostrado ser eficaces en la detección de estresores en diferentes dimensiones, lo que permite abrir líneas de trabajo en función de las carencias descubiertas y así poder corregirlas. Por la actual situación, es recomendable realizar evaluaciones de posibles riesgos psicosociales relacionados, que permita diseñar programas eficaces y que ayuden a una mejor gestión y adaptación en situaciones cambiantes. Con el objetivo de comprender en mayor profundidad los posibles efectos psicológicos de la COVID-19, se recomienda complementar estas herramientas con otras que permitan tener en cuenta nuevas variables como la incertidumbre, la ansiedad o el estado mental y así adecuar tales medidas a la situación real que estamos viviendo (Canepa et al., 2008; Tsutsumi et al.,2001).

\section{REFERENCIAS}

Artazcoz, L., \& Molinero, E. (2004). Evaluación de los factores de riesgo psicosocial combinando metodología cuantitativa y cualitativa. Arch Prev Riesgos Labor, 7(4), 134-142. Recuperado de: https://www.researchgate.net/profile/Lucia Artazcoz/publication/242090729 Evaluaci on de los factores de riesgo psicosocial combinando metodologia cuantitativa y cualitativa/links/0c96052458944bc768000000.pdf

Blanch, J. M., Sahagún, M., \& Cervantes, G. (2010). Estructura factorial del cuestionario de condiciones de trabajo. Revista de Psicología del Trabajo y de las Organizaciones, 26(3), 175-189. Recuperado de: http://scielo.isciii.es/pdf/rpto/v26n3/v26n3a02.pdf

Balbo, L. (1994). La doble presencia. In Las mujeres y el trabajo: rupturas conceptuales (pp. 503-514). Icaria.

Benavides, F. G., Delclós, J., \& Serra, C. (2018). Estado de bienestar y salud pública: el papel de la salud laboral. Gaceta Sanitaria, 32, 377-380. Recuperado de: https://www.scielosp.org/article/gs/2018.v32n4/377-380/

Bourbonnais, R., Comeau, M., \& Vézina, M. (1999). Job strain and evolution of mental health among nurses. Journal of occupational health psychology, 4(2), 95. doi: $\underline{10.1037 / 1076-8998.4 .2 .95}$ 
Riesgos psicosociales y estrés laboral en tiempos de COVID-19: instrumentos para su evaluación

Canepa, C., Briones, J., Pérez, C., Vera, A., \& Juárez, A. (2008). Desequilibrio esfuerzo-recompensa y estado de malestar mental en trabajadores de servicios de salud en Chile. Cien Trab, 10(30), 157-60. Recuperado de: https://www.researchgate.net/profile/Arturo Juarez Garcia/publication/44204847 De sequilibro Esfuerzo-

Recompensa y estado de malestar en Trabajadores de Servicios de Salud en Chile/links/54408de30cf2fd72f99dddba.pdf

Castiblanque, R. P., \& Palomares, R. C. (2020). Culturas organizacionales que refuerzan la intensificación del trabajo a través de recursos digitales y su impacto sobre la salud laboral. Revista Prisma Social, (29), 25-57. Recuperado de: https://dialnet.unirioja.es/servlet/articulo?codigo $=7470983$

Chacín-Almarza, B., Corzo-Alvarez, G., Rojas-González, L., Rodríguez-Chacín, E., \& Corzo-Ríos, G. (2002). Estrés organizacional y exposición a ruido en trabajadores de la planta de envasado de una industria cervecera. Investigación Clínica, 43(4), 271289.

Recuperado

de: https://pdfs.semanticscholar.org/b4c7/d6d7969ed2bb2477cf80b9f2c02318359ea9.pdf

Cigna (2019). Cigna 360 wellbeing survey 2019 - "Well and Beyond". Recuperado de: https://wellbeing.cigna.com/

Cladellas Pros, R. (2008). La ausencia de gestión de tiempo como factor de riesgo psicosocial en el trabajo. Recuperado de: https://upcommons.upc.edu/bitstream/handle/2099/6957/cladellas.pdf?sequence=1\&i sAllowed $=y$

Dawis, R. V., \& Lofquist, L. H. (1984). A psychological theory of work adjustment: An individual-differences model and its applications. University of Minnesota press.

Díaz, J., \& Tinoco, V. (2011). Niveles de estrés laboral en empleados de una institución policial civil de la ciudad de SantaMarta. Duazary, 8(1), 6-12. doi: 10.21676/2389783X

EU-OSHA (2019). Encuesta de la Agencia Europea para la Seguridad y la Salud en el trabajo ESENER 2019 "What does it tell us about safety and health in Europe's workplaces?". Recuperado de: https://osha.europa.eu/es/publications/esener-2019policy-brief/view

EU-OSHA (2020). COVID-19: VUELTA LA TRABAJO - Adaptar los lugares de trabajo para proteger a los trabajadores. Recuperado de: https://osha.europa.eu/es/publications/covid-19-back-workplace-adaptingworkplaces-and-protecting-workers/view

Eurostat (2019). Data recogida por la oficina estadística de la Unión Europea sobre trabajo y salud. Recuperado de: https://appsso.eurostat.ec.europa.eu/nui/show.do?dataset=hsw exp1\&lang=en

Revista de Comunicación y Salud, 2020, Vol. 10, oㅡ 2, pp. 301-321 
Riesgos psicosociales y estrés laboral en tiempos de COVID-19: instrumentos para su evaluación

Fernández-López, J. A., Fernández-Fidalgo, E., y Siegrist, J. (2005). El trabajo y sus repercusiones en la salud. El modelo "Desequilibrio Esfuerzo-Recompensa-DER". Revista de Calidad Asistencial, 20 (3), 165-170. doi: 10.1016/S1134-282X(08)74743-2

Gallegos, W. L. A., Iturrizaga, I. M., \& Salinas, M. A. M. (2014). El modelo demanda control de Karasek y su relación con la creatividad docente en profesores de nivel primario de Arequipa. REVISTA DE PSICOLOGÍA/Journal of Psychology, 16(1), 6477. Recuperado de: http://181.224.246.204/index.php/R PSI/article/view/236/139

García, M. M., Iglesias, S., Saleta, M., \& Romay, J. (2016). Riesgos psicosociales en el profesorado de enseñanza universitaria: diagnóstico y prevención. Revista de Psicología del Trabajo y de las Organizaciones, 32(3), 173-182. doi: $\underline{10.1016 / j . r p t o .2016 .07 .001}$

García-Herrero, S., Mariscal, M. A., Gutiérrez, J. M., \& Ritzel, D. O. (2013). Using Bayesian networks to analyze occupational stress caused by work demands: Preventing stress through social support. Accident Analysis \& Prevention, 57, 114123. doi: $\underline{10.1016 / \text { j.aap.2013.04.009 }}$

García-Rodríguez, A., Gutiérrez-Bedmar, M., Bellón-Saameño, J. Á., Muñoz-Bravo, C., \& Navajas, J. F. C. (2015). Entorno psicosocial y estrés en trabajadores sanitarios de la sanidad pública: diferencias entre atención primaria y hospitalaria. Atención Primaria, 47(6), 359-366. doi: 10.1016/j.aprim.2014.09.003

García, C. C., Rísquez, M. I. R., Fernández, L. E., \& Roche, M. E. M. (2018). Factores de estrés laboral en el personal de enfermería hospitalario del equipo volante según el modelo de demanda-control-apoyo. Enfermería global, 17(2), 304-324. doi: $\underline{10.6018 / \text { eglobal.17.2.277251 }}$

Instituto Nacional de Seguridad e Higiene en el Trabajo [INSHT] (2012). I Encuesta sobre Condiciones de Trabajo y Salud en Iberoamérica (I ECCTS).

Instituto Nacional de Seguridad y Salud en el Trabajo (2020). Prevención de riesgos laborales vs. COVID-19 - Compendio no exhaustivo de fuentes de información. Recuperado de: https://www.insst.es/documents/94886/693030/Prevenci\%C3\%B3n+de+riesgos+labor ales+vs.+COVID-19++Compendio+no+exhaustivo+de+fuentes+de+informaci\%C3\%B3n/4098124f-532443a6-8881-0bbd4e358de7

Ivancevich, J. M., \& Matteson, M. T. (1989). Estrés y trabajo: una perspectiva gerencialStress and work: a managerial perspective (No. 658.38 193l 1989). Trillas. 
Riesgos psicosociales y estrés laboral en tiempos de COVID-19: instrumentos para su evaluación

Jiménez, B. M., \& León, C. B. (2010). Factores y riesgos psicosociales, formas, consecuencias, medidas y buenas prácticas. Universidad Autónoma de Madrid, 19. Recuperado de: https://www.researchgate.net/profile/Bernardo MorenoJimenez/publication/236151656 factores y riesgos psicosociales formas consecue ncias INSHT/links/0deec5166da54c17aa000000.pdf

Johnson, J. V. (1986). The impact of workplace social support, job demands and work control upon cardiovascular disease in Sweden (Doctoral dissertation, ProQuest Information \& Learning).

Johnson, J. V., \& Hall, E. M. (1988). Job strain, work place social support, and cardiovascular disease: a cross-sectional study of a random sample of the Swedish working population. American journal of public health, 78(10), 1336-1342. doi: 10.2105/AJPH.78.10.1336

Karasek Jr, R. A. (1979). Job demands, job decision latitude, and mental strain: Implications for job redesign. Administrative science quarterly, 285-308. doi: $\underline{10.2307 / 2392498}$

Karasek, R., y Theorell, T. (1990). Healthy work: Stress, productivity, and the reconstruction of working life. Nueva York: Basic Books.

Kivimäki, M., Leino-Arjas, P., Luukkonen, R., Riihimäi, H., Vahtera, J., \& Kirjonen, J. (2002). Work stress and risk of cardiovascular mortality: prospective cohort study of industrial employees. Bmj, 325(7369), 857. doi: 10.1136/bmj.325.7369.857

Kristensen, T. S. (2000). A new tool for assessing psychosocial factors at work: The Copenhagen Psychosocial Questionnaire. Copenhagen: National Institute of Health

Landsbergis, P. A., Schnall, P. L., Pickering, T. G., \& Schwartz, J. E. (2002). Validity and reliability of a work history questionnaire derived from the Job Content Questionnaire. Journal of occupational and environmental medicine, 44(11), 1037-1047. doi: $\underline{10.1097 / 00043764-200211000-00010}$

Lazarus, R. S. y Folkman, S. (1986). Estrés y procesos cognitivos. Barcelona: Martínez Roca

Lazcano, L. A., i Cubells, J. C., i Liuís, S. M., \& Miguel, A. S. (1996). Estrés y tensión laboral en enfermeras y auxiliares de clínica de hospital. Gaceta sanitaria, 10(57), 282-292. doi: $\underline{10.1016 / S 0213-9111(96) 71901-5}$

Leka S, Griffiths A, Cox T (2004). La organización del trabajo y el estrés. Serie protección de la salud de los trabajadores $N^{\circ} 3$. OIT- OMS. 2004. Recuperado de: https://www.who.int/occupational health/publications/pwh3sp.pdf 
Riesgos psicosociales y estrés laboral en tiempos de COVID-19: instrumentos para su evaluación

Ley de Prevención de Riesgos Laborales. L BOE núm. 269, 10 de noviembre de1995 (8 noviembre 1995). Recuperado de: https://www.boe.es/buscar/pdf/1995/BOE-A-199524292-consolidado.pdf

López González, A. A., Hidalgo García, M., Martínez Palmer, M. A., Valens Mesquida, M., Suerda Parera, A. M. y Monroy Fuenmayor, N. (2004). Estudio de estrés laboral en Baleares aplicando el cuestionario desequilibrio esfuerzo-recompensa. Medicina Balear, 19 (3), 26-30. Recuperado de https://dialnet.unirioja.es/servlet/articulo?codigo $=6370791$

Lluís, J., \& Navarro, M. (2006). ¿Cómo evaluar los riesgos psicosociales en la empresa? Metodologías, oportunidades y tendencias. Recuperado de: https://www.prevencionintegral.com/canal-orp/papers/orp-2006/como-evaluarriesgos-psicosociales-en-empresa-metodologias-oportunidades-tendencias

Luceño, L., Martín, J., Jaén, M., y Díaz, E. M. (2005). Evaluación de factores psicosociales en el entorno laboral. EduPsykhé, 4 (1), 19-42. Recuperado de: http://repositorio.ucjc.edu/bitstream/handle/20.500.12020/113/C00031080.pdf?seque $\underline{\text { nce }=1}$

Ministerio de trabajo y economía social (2020). Resumen datos estadísticos de empleo en España. Recuperado de: http://www.mitramiss.gob.es/ficheros/ministerio/estadisticas/documentos/RUD.pdf

Moncada, S. (2004). Método istas21 (CoPsoQ): manual para la evaluación de riesgos psicosociales en el trabajo: incluye la adaptación para el Estado español del Cuestionario Psicosocial de Copenhague (CoPsoQ). ISTAS.

Moncada, S., Llorens, C., Navarro, A., \& Kristensen, T. S. (2005). ISTAS21: Versión en lengua castellana del cuestionario psicosocial de Copenhague (COPSOQ). Arch Prev Riesgos Labor, 8(1), 18-29. Recuperado de: https://www.researchgate.net/profile/Salvador Lluis/publication/238752287 ISTAS21 Version en lengua castellana del cuestionario psicosocial de Copenhague CO PSOQ/links/0deec529c4c225a8e8000000.pdf

Moreno, M. P., Beltrán, C. A., Serrano, L. P., Chávez, S. A. F., \& Estrada, J. G. S. (2006). Validez y confiabilidad del inventario de violencia y acoso psicológico en el trabajo (Ivapt-Pando). Enseñanza e investigación en Psicología, 11(2), 319-332. Recuperado de: https://www.redalyc.org/pdf/292/29211208.pdf

Olmos, J. G., Cebollero, M. P., \& Carrejón, M. B. (2008). Propiedades psicométricas de la batería de evaluación de riesgos psicosociales en la mediana y pequeña empresa. Psicothema, 20(4), 939-944. Recuperado de: https://www.redalyc.org/pdf/727/72720465.pdf 
Riesgos psicosociales y estrés laboral en tiempos de COVID-19: instrumentos para su evaluación

Organización Internacional del Trabajo (2019). Informe "Seguridad y salud en el centro del futuro del trabajo". Recuperado de: http://www.medicosypacientes.com/sites/default/files/wcms 686766.pdf

Organización Internacional del Trabajo (2020) "El COVID-19 y el mundo del trabajo. Tercera edición". Recuperado de: https://www.llo.org/wcmsp5/groups/public/--dgreports/---dcomm/documents/briefingnote/wcms 743154.pdf

Organización Mundial de la Salud (1948). Constitución de la Organización Mundial de la Salud. Disponible en: https://pnsd.sanidad.gob.es/pnsd/legislacion/pdfestatal/i5.pdf

Organización Mundial de la Salud (2020). A safe and healthy return to work during the COVID-19 pandemic. Recuperado de: https://www.ilo.org/wcmsp5/groups/public/--ed protect/---protrav/---safework/documents/briefingnote/wcms 745549.pdf

Ortiz, V. G. (2010). Assessment of psychosocial stressors at work: psychometric properties of the Spanish version of the ERI (Effort-Reward Imbalance) Questionnaire in Colombian Workers. Revista de Psicología del Trabajo y de las Organizaciones, 26(2), 147-156. Recuperado de: https://www.redalyc.org/pdf/2313/231316500006.pdf

Peiró, J. M. (2005). Desencadenantes del estrés laboral. Madrid: Pirámide.

Peiró, J. M. y Rodríguez, I. (2008). Estrés laboral, liderazgo y salud organizacional. Papeles del Psicólogo, 29 (1), 68-82. Recuperado de: https://www.redalyc.org/pdf/778/77829109.pdf

Pervin, L. A. (1967). A twenty-college study of studentx college interaction using tape (transactional analysis of personality and environment): Rationale, reliability, and validity. Journal of Educational Psychology, 58(5), 290. doi: 10.1037/h0025011

Peter, R., Geißler, H., \& Siegrist, J. (1998). Associations of effort-reward imbalance at work and reported symptoms in different groups of male and female public transport workers. Stress medicine, 14(3), 175-182. doi: 10.1002/(SICl)10991700(199807)14:3<175::AID-SMI775>3.0.CO;2-4

Sandín, B. (2003). El estrés: un análisis basado en el papel de los factores sociales. Revista Internacional de Psicología Clínica y de la Salud, 3 (1), 141-157. Recuperado de: https://www.redalyc.org/pdf/337/33730109.pdf

Sauter, S., Murphy, L., Hurrell, J., \& Levi, L. (1998). Factores de riesgos psicosociales y de organización. OIT, Enciclopeida de salud y seguridad en el trabajo. España: Ministerio del trabajo y asuntos sociales.

Selye, H. (1960). La tensión en la vida. Cía. General Fabril. 
Riesgos psicosociales y estrés laboral en tiempos de COVID-19: instrumentos para su evaluación

Siegrist, J. (1996). Adverse health effects of high-effort/low-reward conditions. Journal of Occupational Health Psychology, 1(1), 27-41. doi: 10.1037/1076-8998.1.1.27

Siegrist, J. (2010). Effort-reward imbalance at work and cardiovascular diseases. International journal of occupational medicine and environmental health, 23(3), 279.2 Recuperado de: http://test.imp.lodz.pl/upload/oficyna/artykuly/pdf/full/2010/0\%20Siegrist.pdf

Siegrist, J., Li, J. y Montano, D. (2014). Psychometric properties of the Effort-Reward Imbalance Questionnaire. Dusseldorf: Dusseldorf University. Recuperado de: http://www.uniklinikduesseldorf.de/fileadmin/Datenpool/einrichtungen/institut fuer me dizinische soz iologie id54/ERI/PsychometricProperties.pdf

Sjöberg, O. (2017). Positive welfare state dynamics? Sickness benefits and sickness absence in Europe 1997-2011. Social Science \& Medicine, 177, 158-168. doi: 10.1016/j.socscimed.2017.01.042

Tejada, P. A., \& Gómez, V. (2009). Factores psicosociales y laborales asociados al burnout de psiquiatras en Colombia. Revista colombiana de psiquiatría, 38(3), 488512. Recuperado de: https://www.redalyc.org/pdf/806/80615422008.pdf

Tesorería General de la Seguridad Social (2020). Datos afiliación seguridad social abril 2020. Recuperado de: https://revista.seg-social.es/wpcontent/uploads/2020/05/AFILIACI\%C3\%93N-ABRIL.pdf

Tirado, G., Llorente-Alonso, M., \& Topa, G. (2019). Desequilibrio esfuerzo-recompensa y quejas subjetivas de salud: Estudio exploratorio entre médicos en España. European Journal of Investigation in Health, Psychology and Education, 9(2), 59-70. doi: $10.30552 /$ ejihpe.v9i2.320

Tsutsumi, A., Kayaba, K., Theorell, T., \& Siegrist, J. (2001). Association between job stress and depression among Japanese employees threatened by job loss in a comparison between two complementary job-stress models. Scandinavian journal of work, environment \& health, 146-153. Recuperado de: https://www.jstor.org/stable/40967130?seq=1\#metadata info tab contents

Tunanñaña, A. S. (2013). Adaptación de la escala de estrés laboral de la OIT-OMS en trabajadores de 25 a 35 años de edad de un contact center de ima. PsiqueMag, 2(1).

Uribe-Prado, J. F. (2010). EDO Escala de Desgaste Ocupacional (Burnout). México: Ed. Manual Moderno. Recuperado de: https://pseaconsultores.com/sites/default/files/EDO.pdf

Villalobos, G., Vargas, A. M., Escobar, J., Jiménez, M. L., \& Rondón, M. A. (2010). Batería de instrumentos para la evaluación de factores de riesgo psicosocial. Bogotá: Ministerio de la Protección Social. Recuperado de https://portal.posipedia.co/wpcontent/uploads/2019/08/bateria-instrumento-evaluacion-factores-riesgopsicosocial.pdf 
Riesgos psicosociales y estrés laboral en tiempos de COVID-19: instrumentos para su evaluación

\section{AUTORA}

\section{Luz Martínez-Martínez}

Doctora en Comunicación. Profesora Departamento de Sociología y Comunicación (URJC) y Departamento de Teoría y Análisis de la Comunicación (UCM). Coordinadora laboratorio de neurocomunicación Neurolabcenter de la UCM. Profesora de varios Máster de la UCM y talleres y seminarios de neuromarketing. Perteneciente a grupos de investigación en el ámbito de la comunicación de la UCM y URJC. Participación en numerosos congresos internacionales y autora de diversos artículos en revistas de impacto y capítulos de libro. Participante de proyectos de investigación sobre comportamiento, neurociencia y salud. Líneas de investigación: comunicación, aprendizaje, salud, persuasión y neurociencia. En el campo profesional se ha dedicado siempre al mundo de la comunicación y el marketing como directora de comunicación, desarrollo corporativo, producción y gestión publicitaria.

luzmar03@ucm.es

ORCID ID: http://orcid.org/0000-0001-8582-724X

Google Scholar: https://scholar.google.es/citations?user=YXRnXWMAAAAJ\&hl=es

ResearchGate: https://www.researchgate.net/profile/Luz Martinez Martinez 